\title{
Can Lymphovascular and Perineural Invasion be Additional Staging Criteria in Colorectal Cancer?
}

\author{
Tayfun Kaya and Ayberk Dursun \\ Department of General Surgery, University of Health Sciences, Tepecik Training and Research Hospital, Izmir, Turkey
}

\begin{abstract}
Objective: To identify additional staging information, venous, lymphatic, and neural invasion as potential prognostic factors in colorectal cancer (CRC).

Study Design: A descriptive study.

Place and Duration of the Study: University of Health Sciences, Tepecik Training and Research Hospital, Izmir, Turkey; from May 2007 to June 2019.

Methodology: Retrospective analyses were performed on 855 CRC patients, who were treated with surgery. Patient and treatment characteristics, lymphovascular (LVI), and perineural (PNI) invasion were documented. The impact of LVI and PNI was determined using Cox proportional hazards model.

Results: The cohort examined had 346 (40.5\%) LVI and 150 (17.5\%) PNI positive patients. After surgery, mortality was $18.4 \%$ for $\mathrm{LVI}$ and $8 \%$ for PNI patients. Although increased ASA score (for ASA 2 hazard ratio $[H R]=0.555, p=0.001$ and ASA 3-4 $H R=0.723, p=0.014)$, adjuvant chemotherapy ( $H R=2.5, p<0.001)$, LVI $(H R=1.961, p<0.001)$ and PNI $(H R=1.625$, $\mathrm{p}<0.001$ ) involvement increased the risk of death based on univariate analysis, multivariate Cox analysis showed a risk of death increase with increased ASA score (for ASA $2 \mathrm{HR}=0.53, \mathrm{p}<0.001$ and ASA 3-4 HR=0.703, $\mathrm{p}=0.008$ ), adjuvant chemotherapy ( $H R=2.114, p<0.001)$ and $L V I$ involvement $(H R=1.640, p<0.001)$.

Conclusion: LVI and PNI may be useful in identifying CRC patients who might benefit the most from adjuvant systemic therapy. On the other hand, the presence of LVI and PNI reflects a shorter patient survival.
\end{abstract}

Key Words: Lymphovascular invasion, Perineural invasion, Colorectal cancer, Cancer staging.

How to cite this article: Kaya T, Dursun A. Can Lymphovascular and Perineural Invasion be Additional Staging Criteria in Colorectal Cancer?. J Coll Physicians Surg Pak 2021; 31(06):657-662.

\section{INTRODUCTION}

Colorectal cancer (CRC) is a disease that is usually encountered in the elderly population and is accompanied by unavoidably high morbidity and mortality. The incidence of CRC has been growing fast and is expected to increase by $60 \%$ by $2030 .{ }^{1}$ Worldwide, CRC accounts for $9.4 \%$ of all incident cancer in men and $10.1 \%$ in women with adenocarcinoma being the most common type of CRC observed. ${ }^{2,3}$ Besides, CRC is the second most common cause of cancer-related death in both men and women. ${ }^{2,4}$

Although recent advances in CRC treatment have improved patient prognosis and nearly $65 \%$ of patients attain 5 -year overall survival in high-income countries, this parameter has remained below $50 \%$ in low-income countries. ${ }^{5}$

Correspondence to: Dr. Tayfun Kaya, Department of General Surgery, University of Health Sciences, Tepecik Training and Research Hospital, Izmir, Turkey

E-mail: kayatayfun@yahoo.com

Received: March 28, 2021; Revised: April 28, 2021;

Accepted: May 18, 2021

DOI: https://doi.org/10.29271/jcpsp.2021.06.657
Many recent studies have reported that the stage at diagnosis was the most important prognostic factor in CRC patients. Brenner et al. reported that the 5-year relative survival of patients in the USA diagnosed with localised CRC was $90.1 \%$, with regional spread CRC was $69.2 \%$, and with distant tumor spread was $11.7 \%$. ${ }^{5}$ The localised spread of the tumor is mediated by the invasion of tumor cells; this is an important feature of solid tumors and is indicative of a poor prognosis. ${ }^{6}$ Lymphovascular invasion (LVI) and perineural invasion (PNI) caused by localized tumor spread are considered poor prognostic criteria and affect treatment plans. However, LVI and PNI are not included in classifications.

In recent years, PNI has increasing ly been considered to be relevant to the prognosis of CRC. The infiltration of cancer cells into nerve tissue not only provides a new pathway for metastasis but can biologically alter tumor behavior by providing a new environment fortumor cells. ${ }^{7}$ Recent data concerning the prognostic significance of PNI on patients with CRC are conflicting, and the risk associated with PNI is currently unclear. ${ }^{6-8}$

The aim of this study was to investigate the relationship between LVI and PNI and overall survival in CRC, to examine 
the clinical implications of LVI and PNI on outcomes, and to identify risk factors for poor prognosis in CRC patients.

\section{METHODOLOGY}

Retrospective analyses were performed on 855 CRC patients, surgically treated between May 2007 and June 2019. Demographic features of the patient population, carcinoembryonic antigen (CEA) levels, tumor features, administered treatment and surgical procedures as well as tumor classifications according to survival status, were evaluated. Patients' diagnosis, tumor subtype, histologic differentiation, and presence of lymphovascular invasion (LVI) or PNI were evaluated by histopathology after surgery. The histologic differentiation subtypes (none, cancer-in-situ, well-, moderate-, and poorly-differentiated) and American Joint Committee on Cancer (AJCC) staging system were evaluated according to survival status, PNI, and LVI of the patients.

The findings were analysed with patients categorised according to survival status, histologic differentiation, and AJCC staging system. The endpoint of this study was overall survival (OS), which was defined as the time from surgery to the date of last follow-up or death from any cause.

Software package SPSS for Windows version 25.0 (SPSS, Chicago, IL, USA) was used for statistical analyses. Descriptive statistics included mean and standard deviation. Crosstables were reported as percent ratio. Variables with continuous data were first ascertained for normality of distribution by the shape of the distribution pattern in the Kolmogorov-Smirnov test. The data were then statistically compared using independent sample t test for normally distributed samples. Variables with categorical data were compared using Chi-square test. Survival rates were assessed using the Kaplan-Meier method and compared with the log-rank test. The Cox proportional hazard model used to calculate the hazard ratio (HR) and $95 \%$ confidence interval $(\mathrm{Cl})$. Variables with $p<0.1$ in the univariate analysis were entered into a multivariate Cox analysis with stepwise backward selection. Two-sided $p$ value below 0.05 was considered to be statistically significant.

\section{RESULTS}

Table 1 summarises demographic and clinic pathologic characteristics of the patients ( 518 males, 337 females; mean age $62 \pm$ 12 years, range 18 to 96 years) due to survival status. Overall mortality rate was $34.5 \%(n=295)$. The ratio of patients with both LVI positivity and mortality among all patients was $18.4 \%$, while the ratio of $L V I$ negative survivors among all patients was $43.4 \%(p<0.001)$. Similarly, the ratio of patients with both PNI positivity and mortality among all patients was $8.0 \%$, while the ratio of LVI negative survivors among all patients was $55.9 \%$ $(p=0.002)$. The mortality rate was seen to increase statistically significantly with worsening histological differentiation and in IIIB, IIIC, and IVA stages of the AJCC staging system.

Analyses of demographic, clinicopathologic, and tumor classification characteristics of the patients according to LVI and PNI are shown in Table II. Age, gender, and the ASA score did not show any statistically significant difference based on LVI and PNI. In patients undergoing adjuvant chemotherapy $(n=576)$, LVI positivity was $47.7 \%$ and PNI positivity was $21.2 \%$, which was statistically significant $(p<0.05)$. In patients undergoing neoadjuvant chemoradiotherapy $(n=139)$, LVI positivity was $20.9 \%$ and negativity was $79.1 \%$; while PNI positivity was $16.5 \%$, and negativity was $83.5 \%$. This difference was statistically significant for LVI $(p<0.001)$; but not for PNI $(p=0.736)$. LVI positivity was detected in $53.2 \%$ of patients with mortality $(p<0.001)$, and PNI positivity was $23.1 \%$ for patients with mortality $(p=0.002)$.

The univariate and multivariate Cox regression analyses for overall survival are presented in Table III. Univariate and multivariate analyses and Kaplan-Meier survival plots showed no significant difference between patients who were grouped as youngerand olderthan 65 years. A higher ASA score was associated with a higher risk of death and statistically significant differences in OS were found between ASA subgroups. Patients who underwent $A C$ had a greater risk of death and showed a significantly lower OS. The univariate analysis also showed that LVI and PNI were statistically significant risk factors. The LVI positive group showed a significantly lower OS, like PNI positive group. In the multivariate analysis, the variables associated with poor OS were increased ASA score, presence of LVI and undergoing AC. However, age and PNI did not show statistical significance in the multivariate analysis (Figure 1).

\section{DISCUSSION}

The aim of the current study was to investigate the prognostic significance of PNI in a population of CRC patients who underwent curative surgical resection, to evaluate the significance of PNI on clinical outcomes, and to identify risk factors leading to poor prognosis. The study results revealed that high ASA score, presence of $\mathrm{LVI}$, and undergoing $\mathrm{AC}$ were the significant risk factors for mortality; moreover, these factors had a significant effect on OS. Although univariate analysis indicated the presence of PNI as a risk factor and showed a lower OS rate in a Cox hazard model, multivariate analysis did notsupport this data.

Early diagnosis, good tumor staging, as well as successful surgical and medical interventions are the cornerstones for improved outcomes in CRC patients. ${ }^{1}$ Primary tumor staging is one of the most important steps in determining treatment strategy and predicting the prognosis of the disease in CRC patients. ${ }^{9}$ The classification of the AJCC, according to the TNM stage, provides valuable prognostic information and guides therapy decisions. ${ }^{5,10}$ Its success in predicting prognosis has been found to be effective in early and late stage patients; however, success in prognosis prediction is reported to be lower for intermediate-stage CRC patients. ${ }^{2,3}$ Due to the inherent complications in intermediate-stage $\mathrm{CRC}$, among the parameters of the currently used TNM staging system of AJCC, the depth of local invasion and anatomic extent of CRC at diagnosis have become the most important factors for predicting survival. ${ }^{10}$ 
Table I: Demographic and clinicopathologic characteristics of the patients and analyses for survival status ( $n=855$ ).

\begin{tabular}{|c|c|c|c|c|}
\hline \multirow{2}{*}{\multicolumn{2}{|c|}{ Demographic and clinicopathologic characteristics }} & \multicolumn{3}{|c|}{ Survival status } \\
\hline & & \multirow{3}{*}{\begin{tabular}{l|} 
Exitus \\
$182(21.3)$ \\
$113(13.2)$
\end{tabular}} & \multirow{3}{*}{\begin{tabular}{|l|} 
Alive \\
$323(37.8)$ \\
$237(27.7)$
\end{tabular}} & \multirow{3}{*}{0.256} \\
\hline \multirow{2}{*}{ Age, $n(\%)$} & $<65$ & & & \\
\hline & $>65$ & & & \\
\hline \multirow{2}{*}{ Gender, n (\%) } & Female & $118(13.8)$ & $219(25.6)$ & \multirow{2}{*}{0.800} \\
\hline & Male & $177(20.7)$ & $341(39.9)$ & \\
\hline \multirow{3}{*}{ Adjuvant chemotherapy, n (\%) } & Early mortality & $33(3.9)$ & $0(0 \%)$ & \multirow{3}{*}{$<0.001$} \\
\hline & Performed & $203(23.7)$ & $373(43.6)$ & \\
\hline & Not Performed & $59(6.9)$ & $187(21.9)$ & \\
\hline \multicolumn{2}{|l|}{ Neoadjuvant Chemoradiotherapy, n (\%) Performed } & $39(4.6)$ & $100(11.7)$ & 0.081 \\
\hline \multirow{3}{*}{ American Society of Anesthesiologists Score, n (\%) } & 1 & $46(5.4)$ & $121(14.2)$ & \multirow{3}{*}{0.003} \\
\hline & 2 & $157(18.4)$ & $319(37.3)$ & \\
\hline & $3-4$ & $92(10.8)$ & $120(14)$ & \\
\hline \multirow{3}{*}{ Carcinoembryonic Antigen groups, n (\%) } & $<5$ & $152(18.8)$ & $378(46.7)$ & \multirow{3}{*}{$<0.001$} \\
\hline & $5-10$ & $40(4.9)$ & $83(10.2)$ & \\
\hline & $>10$ & $79(9.8)$ & $78(9.6)$ & \\
\hline \multirow{2}{*}{ Lymphovascular invasion, n (\%) } & Positive & $157(18.4)$ & $189(22.1)$ & \multirow{2}{*}{$<0.001$} \\
\hline & Negative & $138(16.1)$ & $371(43.4)$ & \\
\hline \multirow{2}{*}{ Perineural invasion, n (\%) } & Positive & $68(8.0)$ & $82(9.6)$ & \multirow{2}{*}{0.002} \\
\hline & Negative & $227(26.5)$ & $478(55.9)$ & \\
\hline \multirow{5}{*}{$\begin{array}{l}\text { Histologic differentiation, } \\
\text { n (\%) }\end{array}$} & None & $11(1.3)$ & $31(3.6)$ & \multirow{5}{*}{0.013} \\
\hline & Cancer-in-situ & $1(0.1)$ & $13(1.5)$ & \\
\hline & Well-differentiated & $20(2.3)$ & $66(7.7)$ & \\
\hline & Moderately-differentiated & $245(28.7)$ & $422(49.4)$ & \\
\hline & Poorly-differentiated & $18(2.1)$ & $28(3.3)$ & \\
\hline \multirow{11}{*}{$\begin{array}{l}\text { The American Joint Committee on Cancer staging classification, } \\
\text { n (\%) }\end{array}$} & 0 & $8(1.0)$ & $26(3.0)$ & \multirow{11}{*}{$<0.001$} \\
\hline & I & $23(2.7)$ & $84(9.8)$ & \\
\hline & IIA & $56(6.5)$ & $179(20.9)$ & \\
\hline & IIB & $28(3.3)$ & $49(5.7)$ & \\
\hline & IIC & $7(0.8)$ & $16(1.9)$ & \\
\hline & IIIA & $4(0.5)$ & $23(2.7)$ & \\
\hline & IIIB & $83(9.7)$ & $132(15.4)$ & \\
\hline & IIIC & $40(4.7)$ & $30(3.5)$ & \\
\hline & IVA & $39(4.6)$ & $17(2)$ & \\
\hline & IVB & $1(0.1)$ & $1(0.1)$ & \\
\hline & IVC & $6(0.7)$ & $3(0.4)$ & \\
\hline
\end{tabular}

Despite advances in molecular and genetic markers that support staging systems, the presence of lymphovascular invasion (LVI) or perineural invasion (PNI) is not addressed in the T staging of TNM and is also not regarded as a prognostic factor that may affect the care of the patient.,10 Many studies have indicated that the presence of LVI or PNI has significant negative effects on prognosis in CRC. ${ }^{6,7}$ By definition, detection of $\mathrm{LVI}$, which refers to the invasion of tumor cells to the lymphatic or blood vessel wall by crossing the full fold, or PNI which refers to the growth of tumor in, around, and through nerves and nerve sheaths, suggests that the metastatic process has begun. As the tumor is no longer localised, LVI and PNI are likely to have a direct relationship with survival and recurrence. ${ }^{1,2,10,11}$ In the decision for chemotherapy, radiotherapy, and surgical options, it is highly important to identify low risk patients who will do well with minimal intervention and those patients who have poor prognosis that need radical surgery and adjuvant treatments. ${ }^{2}$ The literature highlights the importance of LVI and PNI in the evaluation of survival in CRC; however, there is currently no consensus on their unification with staging and decisions regarding treatment options. ${ }^{2,11}$

The most important characteristic of malignant cells is their ability to separate from the primary tumor and disseminate into the systemic circulation leading to metastasis. Metastasis is the primary factor that causes tumor recurrence and is associated with a poor prognosis., ${ }^{4,12}$ LVI has been ascertained in many studies as a poor prognostic factor with suggestions to add the assessment of LVI to the current TNM staging system. ${ }^{4,13,14}$ In the present study, light microscopy assessment indicated that LVI and PNI were found in CRC patients with post-treatment mortality, which was comparable with previously reported findings. ${ }^{4}$ Additionally, the present study showed a significant correlation between increased serum levels of CEA, increased histological tumor grade, advanced tumor stage, and the presence of LVI and PNI. Both LVI and PNI are considered as indicators of the depth of invasion and spread to regional lymph nodes, and the presence of either condition may be considered as evidence of a more aggressive tumor. ${ }^{5,7,15}$

Similar to LVI, PNI is associated with metastatic progress, and is considered a poor prognostic factor in the literature..$^{2,16-19}$ In a review article, the process of PNI occurs by reciprocal signalling between tumor cells and nerves in which neurotrophic growth factors that are recognised as neurotrophins, play a major role. ${ }^{7}$ Neurotrophins are considered as a therapeutic target because of the increased expression of neurotrophins by tumor cells is likely to be an escape mechanism from dependence on paracrine expression by stromal elements. ${ }^{7,18}$ 
Table II. Demographic, clinicopathologic, and tumor classification characteristics of the patients according to lymphovascular invasion and perineural invasion $(n=855)$.

\begin{tabular}{|c|c|c|c|c|c|c|c|c|}
\hline \multirow{2}{*}{\multicolumn{2}{|c|}{$\begin{array}{l}\text { Demographic, clinicopathologic and tumor } \\
\text { classification characteristics }\end{array}$}} & \multirow{4}{*}{$\begin{array}{l}\text { Total } \\
505(59.1) \\
350(40.9)\end{array}$} & \multicolumn{3}{|c|}{ Lymphovascular invasion } & \multicolumn{3}{|c|}{ Perineural invasion } \\
\hline & & & \multirow{2}{*}{$\begin{array}{l}\text { Positive } \\
215(25.1)\end{array}$} & \multirow{3}{*}{\begin{tabular}{|l|} 
Negative \\
$290(33.9)$ \\
$219(25.6)$ \\
\end{tabular}} & \multirow{3}{*}{\begin{tabular}{|l|}
$\mathbf{p}$ \\
0.132
\end{tabular}} & \multirow{3}{*}{\begin{tabular}{|l|} 
Positive \\
$91(10.6)$ \\
$59(6.9)$
\end{tabular}} & \multirow{3}{*}{\begin{tabular}{l|} 
Negative \\
$414(48.4)$ \\
$291(34)$
\end{tabular}} & \multirow{3}{*}{\begin{tabular}{|l|}
$\mathbf{p}$ \\
0.660
\end{tabular}} \\
\hline \multirow{2}{*}{ Age groups, n (\%) } & $<65$ & & & & & & & \\
\hline & $>65$ & & $131(15.3)$ & & & & & \\
\hline \multirow{2}{*}{ Gender, n (\%) } & Female & $337(39.4)$ & $128(15)$ & $209(24.4)$ & \multirow{2}{*}{0.232} & $54(6.3)$ & $283(33.1)$ & \multirow{2}{*}{0.346} \\
\hline & Male & $518(60.6)$ & $218(25.5)$ & $300(35.1)$ & & $96(11.2)$ & $422(49.4)$ & \\
\hline \multirow{3}{*}{ Adjuvant chemotherapy, n (\%) } & Early mortality & $33(3.9)$ & $18(2.1)$ & $15(1.8)$ & \multirow{3}{*}{$<0.001$} & $9(1.1)$ & $24(2.8)$ & \multirow{3}{*}{$<0.001$} \\
\hline & Performed & $576(67.4)$ & $275(32.2)$ & $301(35.2)$ & & $122(14.3)$ & $454(53.1)$ & \\
\hline & Not Performed & $246(28.8)$ & $53(6.2)$ & $193(22.6)$ & & $19(2.2)$ & $227(26.5)$ & \\
\hline \multicolumn{2}{|c|}{ Neoadjuvant chemoradiotherapy, $\mathrm{n}(\%)$} & $139(16.3)$ & $29(3.4)$ & $110(12.9)$ & $<0.001$ & $23(2.7)$ & $116(13.6)$ & 0.736 \\
\hline \multirow{3}{*}{$\begin{array}{l}\text { American Society of } \\
\text { Anesthesiologists Score, } \\
n(\%)\end{array}$} & 1 & $167(19.5)$ & $70(8.2)$ & $97(11.3)$ & \multirow{3}{*}{0.453} & $26(3)$ & $141(16.5)$ & \multirow{3}{*}{0.755} \\
\hline & 2 & $476(55.7)$ & $198(23.2)$ & $278(32.5)$ & & $86(10.1)$ & $390(45.6)$ & \\
\hline & $3-4$ & $212(24.8)$ & $78(9.1)$ & $134(15.7)$ & & $38(4.4)$ & $174(20.4)$ & \\
\hline \multicolumn{2}{|c|}{ Carcinoembryonic antigen $(\mathrm{ng} / \mathrm{mL})$, Mean \pm SD } & $14.14 \pm 45.60$ & $21.5 \pm 65.71$ & $9.21 \pm 22.94$ & $<0.001$ & $23.57 \pm 54.74$ & $12.16 \pm 43.22$ & 0.007 \\
\hline \multicolumn{2}{|c|}{ Mortality, n (\%) } & $295(34.5)$ & $157(18.4)$ & $138(16.1)$ & $<0.001$ & $68(8.0)$ & $227(26.5)$ & 0.002 \\
\hline \multicolumn{2}{|l|}{ Lymphovascular invasion, n (\%) } & $346(40.5)$ & - & - & - & $107(12.5)$ & $239(28.0)$ & $<0.001$ \\
\hline \multicolumn{2}{|l|}{ Perineural invasion, $\mathrm{n}(\%)$} & $150(17.5)$ & $107(12.5)$ & $43(5.0)$ & $<0.001$ & - & - & - \\
\hline \multirow{5}{*}{$\begin{array}{l}\text { Histologic differentiation, } \\
\text { n (\%) }\end{array}$} & None & $42(4.9)$ & $4(0.5)$ & $38(4.4)$ & \multirow{5}{*}{$<0.001$} & $4(0.5)$ & $38(4.4)$ & \multirow{5}{*}{0.006} \\
\hline & Cancer-in-situ & $14(1.6)$ & $0(0)$ & $14(1.6)$ & & $0(0)$ & $14(1.6)$ & \\
\hline & Well & $86(10.1)$ & $31(3.6)$ & $55(6.4)$ & & $10(1.2)$ & $76(8.9)$ & \\
\hline & Moderately & $667(78.0)$ & $283(33.1)$ & $384(44.9)$ & & $121(14.2)$ & $546(63.9)$ & \\
\hline & Poorly & $46(5.4)$ & $28(3.3)$ & $18(2.1)$ & & $15(1.8)$ & $31(3.6)$ & \\
\hline & 0 & $34(4.0)$ & $4(0.4)$ & $30(3.6)$ & & $3(0.3)$ & $31(3.7)$ & \\
\hline & I & $107(12.5)$ & $10(1.2)$ & $97(11.3)$ & & $5(0.6)$ & $102(11.9)$ & \\
\hline & IIA & $235(27.5)$ & $69(8.1)$ & $166(19.4)$ & & $26(3)$ & $209(24.4)$ & \\
\hline & IIB & $77(9.0)$ & $20(2.3)$ & $57(6.7)$ & & $12(1.4)$ & $65(7.6)$ & \\
\hline & IIC & $23(2.7)$ & $10(1.2)$ & $13(1.5)$ & & $3(0.4)$ & $20(2.3)$ & \\
\hline on Cancer Staging System, & IIIA & $27(3.2)$ & $12(1.4)$ & $15(1.8)$ & $<0.001$ & $2(0.2)$ & $25(2.9)$ & $<0.001$ \\
\hline n (\%) & IIIB & $215(25.1)$ & $120(14)$ & $95(11.1)$ & & $51(6.0)$ & $164(19.1)$ & \\
\hline & IIIC & $70(8.2)$ & $58(6.8)$ & $12(1.4)$ & & $29(3.4)$ & $41(4.8)$ & \\
\hline & IVA & $56(6.5)$ & $35(4.1)$ & $21(2.5)$ & & $16(1.9)$ & $40(4.7)$ & \\
\hline & IVB & $2(0.2)$ & $2(0.2)$ & $0(0)$ & & $1(0.1)$ & $1(0.1)$ & \\
\hline & IVC & $9(1.1)$ & $6(0.7)$ & $3(0.4)$ & & $2(0.2)$ & $7(0.8)$ & \\
\hline
\end{tabular}

Table III: Univariate and multivariate Cox regression analyses for overall survival.

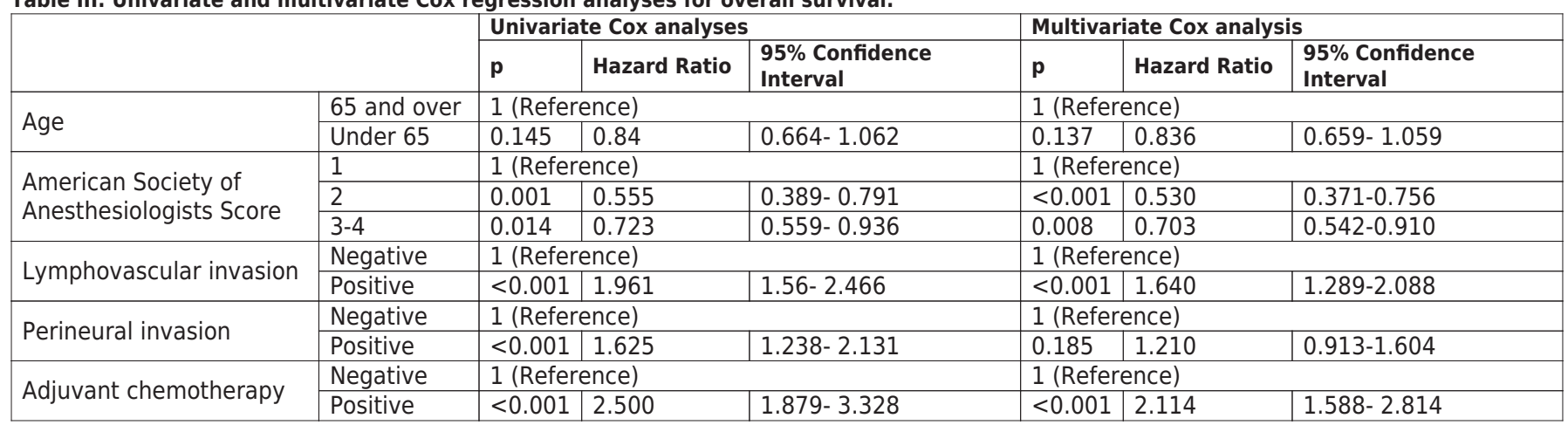

Furuta et al. reported that signalling through the GDNF receptor via increased integrin expression can affect the adhesion of cancer cells to the extracellular matrix (ECM) proteins and invasion in CRC. ${ }^{18}$ It is also reported that tumor cells express matrix metalloproteinases for migration through the ECM.

As further studies illuminate the mechanisms for the metastatic processes in LVI and PNI, future therapeutic strategies targeting these processes may inhibit the metastatic spread of cancer and prolong the survival of patients with $\mathrm{CRC}^{2,4,7,20}$
Firstly, the current study has a retrospective design and consists of patients' data from a single centre. Secondly, several or potentially relevant variables and outcome measures (e.g., local and systemic recurrence), were not available or were incompletely reported in the patient database and, therefore, could not be considered in the analysis. Those patients whose surgical treatment was performed in the clinic where the study was conducted but other cancer treatments were performed at other centres, or those patients with missing information, were excluded from the study. The authors did not have access to data on baseline functional status, which strongly influences the treatment decision-making process and outcomes for cancer 
patients. There was no access to information on specific perioperative complications, chemotherapy regimen, toxicity profile, and the number of treatment cycles received by the subjects. Despite the noted limitations, this study contributes towards understanding the short- and long-term prognostic implications of PNI and LVI in patients with CRC.

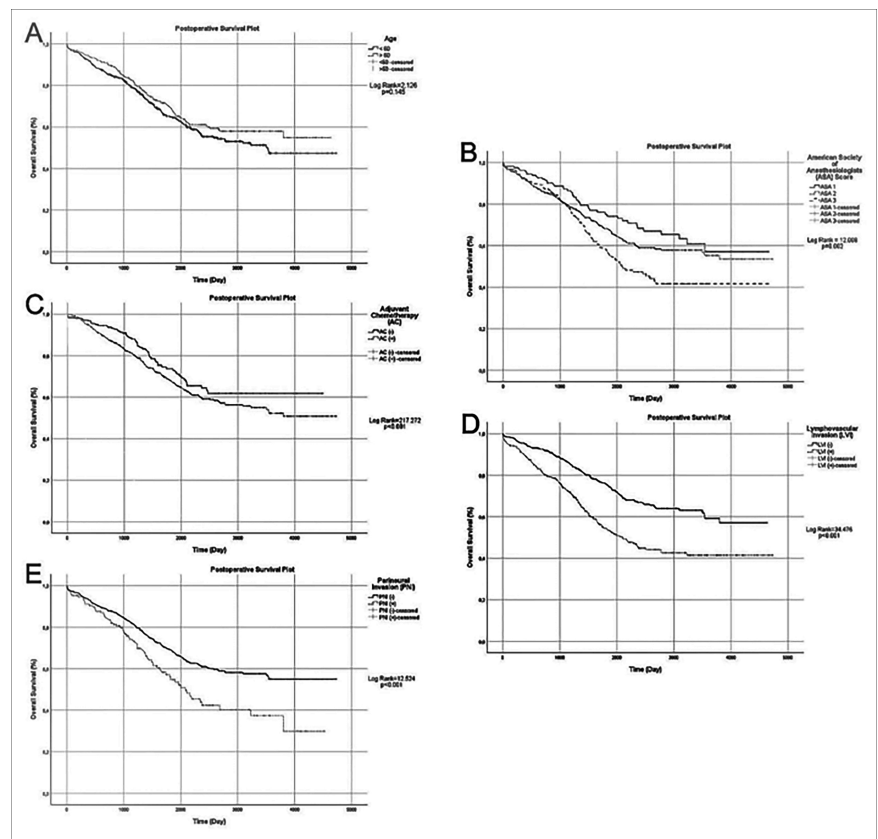

Figure 1. Cox regression analyses of overall survival after primer tumor resection of the colorectal carcinoma based on age (A), ASA score (B), adjuvant chemotherapy (C), histological lymphovascular invasion (LVI) (D) and perineural invasion (PNI) (E).

\section{CONCLUSION}

The results of this study support recent data on the prognostic significance of LVI and PNI that may provide a personalised estimate of prognosis as a guide for managing the treatment. Moreover, these findings will make a positive contribution and better clarify the role of LVI and PNI on the prognostication of CRC patients. Consequently, in the future, LVI and PNI may be added as criteria for staging CRC.

\section{ETHICAL APPROVAL:}

The study protocol was approved by the Ethics Committee of Tepecik Training and Research Hospital, Izmir, Turkey (Decision No. 2020/ 8-8).

\section{PATIENTS' CONSENT:}

Informed consents were obtained from all patients to publish the data concerning this study.

\section{CONFLICT OF INTEREST:}

The authors declared no conflict of interest.

\section{AUTHORS' CONTRIBUTION:}

TK, AD: Conception and design, data collection, analysis and interpretation of data, literature search, drafting of the manuscript, writing and critical review, and final approval. Agreed to be responsible for all aspects of the study in ensuring that questions regarding the accuracy or completeness of any part of the study are properly investigated and resolved.

\section{REFERENCES}

1. Koncina E, Haan S, Rauh S, Letellier E. Prognostic and Predictive Molecular Biomarkers for Colorectal Cancer: Updates and Challenges. Cancers (Basel) 2020; 12(2):319. doi: 10.3390/cancers12020319.

2. Al-Sukhni E, Attwood K, Gabriel EM, LeVea CM, Kanehira K, Nurkin SJ. Lymphovascular and perineural invasion are associated with poor prognostic features and outcomes in colorectal cancer: A retrospective cohort study. Int J Surg 2017; 37:42-9. doi: 10.1016/j.ijsu.2016.08.528.

3. Boyle P, Langman JS. Epidemiology. BMJ 2000; 321 (7264):805.

4. Zhong JW, Yang SX, Chen RP, Zhou YH, Ye MS, Miao L, et al. Prognostic value of lymphovascular invasion in patients with stage III colorectal cancer: A retrospective study. Med Sci Monit 2019; 25:6043-50. doi: 10.12659/MSM.918133.

5. Brenner H, Kloor M, Pox CP. Colorectal cancer. Lancet 2014; 383(9927): 1490-502.

6. Hu G, Li L, Hu K. Clinical implications of perineural invasion in patients with colorectal cancer. Medicine (Baltimore) 2020; 99(17):e19860. doi: 10.1097/MD.000000000001 9860.

7. Liebig C, Ayala G, Wilks JA, Berger DH, Albo D. Perineural invasion in cancer: A review of the literature. Cancer 2009; 115(15):3379-91. doi: 10.1002/cncr.24396.

8. Sun Q, Liu T, Liu P, Luo J, Zhang N, Lu K, et al. Perineural and lymphovascular invasion predicts for poor prognosis in locally advanced rectal cancer after neoadjuvant chemoradiotherapy and surgery. J Cancer 2019; 10(10): 2243-9. doi: 10.7150/jca.31473.

9. Benson $A B$, Schrag D, Somerfield MR, Cohen AM, Figueredo $A T$, Flynn PJ, et al. American Society of Clinical Oncology recommendations on adjuvant chemotherapy for stage II colon cancer. J Clin Oncol 2004; 22(16):3408-19. doi: 10.1200/JCO.2004.05.063.

10. Kim CH, Yeom SS, Lee SY, Kim HR, Kim YJ, Lee KH, et al. Prognostic impact of perineural invasion in rectal cancer after neoadjuvant chemoradiotherapy. World J Surg 2019; 43(1):26-72. doi: 10.1007/s00268-018-4774-8.

11. Bozkurt O, Firat ST, Dogan E. The impact of lymphovascular invasion on recurrence-free survival in high-risk stage II colorectal cancer patients treated with adjuvant therapy. Erciyes Med J 2019; 41(2):191-5.

12. Chablani $P$, Nguyen $P$, Pan $X$, Robinson $A$, Walston $S$, Wu $C$, et al. Perineural invasion predicts for distant metastasis in locally advanced rectal cancer treated with neoadjuvant chemoradiation and surgery. Am J Clin Oncol 2017; 40(6):561-8. doi: 10.1097/COC.0000000000000214.

13. Huh JW, Kim HR, Kim YJ. Lymphovascular or perineural invasion may predict lymph node metastasis in patients with $\mathrm{T} 1$ and T2 colorectal cancer. J Gastrointest Surg 2010; 14(7):1074-80. doi: 10.1007/s11605-010-1206-y. 
14. Barresi V, Reggiani Bonetti L, Vitarelli E, Di Gregorio C, Ponz de Leon M, Barresi G. Immunohistochemical assessment of lymphovascular invasion in stage I colorectal carcinoma: Prognostic relevance and correlation with nodal micrometastases. Am J Surg Pathol 2012; 36(1):66-72. doi: 10.1097/PAS.0b013e31822d3008.

15. Yuan H, Dong Q, Zheng B, Hu X, Xu JB, Tu S. Lymphovascular invasion is a high risk factor for stage $1 /$ II colorectal cancer: A systematic review and meta-analysis. Oncotarget 2017; 8(28):46565-79. doi: 10.18632/onco target.15425.

16. Yang $Y$, Huang $X$, Sun J, Gao $P$, Song $Y$, Chen $X$, et al. Prognostic value of perineural invasion in colorectal cancer: $A$ meta-analysis. J Gastrointest Surg 2015; 19(6):1113-22. doi: 10.1007/s11605-015-2761-z.

17. Knijn N, Mogk SC, Teerenstra S, Simmer F, Nagtegaal ID. Perineural invasion is a strong prognostic factor in colorectal cancer: A systematic review. Am J Surg Pathol
2016; 40(1):103-12. doi: 10.1097/PAS.0000000000000 518.

18. Furuta A, Funahashi H, Sawai H, Sato M, Okada $Y$, Takeyama $\mathrm{H}$, et al. The relationship between GDNF and integrins in human colorectal cancer cell activity. Hepatogastroenterology.2007; 54(77):1398-402.

19. Stojkovic Lalosevic M, Milovanovic T, Micev M, Stojkovic M, Dragasevic $S$, Stulic $M$, et al. Perineural invasion as a prognostic factor in patients with stage I-III rectal cancer - 5-year follow up. World J Gastrointest Oncol 2020; 12(5):592-600. doi: 10.4251/wjgo.v12.i5.592.

20. Pricolo VE, Steingrimsson J, McDuffie TJ, McHale JM, McMillen B, Shparber M. Tumor Deposits in Stage III Colon Cancer: Correlation With Other Histopathologic Variables, Prognostic Value, and Risk Stratification-Time to Consider "N2c". Am J Clin Oncol 2020; 43(2):133-8. doi: 10.1097/ COC.00000000 00000645 . 